\title{
MicroRNA 1283 alleviates cardiomyocyte damage caused by hypoxia / reoxygenation via targeting GADD $45 A$ and inactivating the JNK and p38 MAPK signaling pathways
}

\author{
Chongtao Liu¹, Hongwei Liu², Quan Sun², Pengxia Zhang¹ \\ 1 Department of Biochemistry and Molecular Biology, School of Basic Medicine, Jiamusi University, Jiamusi, Heilongjiang, China \\ 2 Department of Biochemistry and Molecular Biology, Jiamusi University, Jiamusi, Heilongjiang, China
}

\section{KEY WORDS}

apoptosis, cell viability, GADD45A, miR-1283
Correspondence to: Prof. Pengxia Zhang, Department of Biochemistry and Molecular Biology, School of Basic Medicine, Jiamusi University, No. 148 Xuefu Road, Jiamusi, Heilongjiang 154007, China, phone: +8604548618518, email: pengxiaz@126.com Received: June 28, 2020 Revision accepted: November 18, 2020 Published online: December 3, 2020 Kardiol Pol. 2021; 79 (2): 147-155 doi:10.33963/KP.15696 Copyright by the Author(s), 2021

\section{ABSTRACT}

BACKGROUND Clarifying the molecular mechanism and identifying markers of myocardial ischemia/reperfusion injury is crucial for the treatment of acute myocardial infarction.

AIMS This study aimed to investigate the roles and underlying regulatory mechanisms of microRNA 1283 (miR-1283) and GADD45A in cardiomyocytes injured by hypoxia / reoxygenation (H/R).

METHODS Bioinformatic analyses were used to determine the expression of GADD45A and miR-1283 based on various datasets from the Gene Expression Omnibus database. Human embryonic cardiomyocytes were subjected to $\mathrm{H} / \mathrm{R}$ to construct in vitro models. Real-time quantitative polymerase chain reaction and Western blot were used to detect mRNA and protein expression levels, respectively. The binding sites between miR-1283 and GADD45A were predicted by the TargetScan software and verified using dual luciferase reporter assays. Cell viability and apoptosis were detected with the use of Cell Counting Kit 8 and flow cytometry assays.

RESULTS GADD45A and miR-1283 were upregulated or downregulated in myocardial infarction, respectively. MicroRNA 1283 expression was decreased in cardiomyocytes after $\mathrm{H} / \mathrm{R}$ treatment. $\mathrm{H} / \mathrm{R}$ treatment reduced cardiomyocyte viability and enhanced apoptosis, and these effects were abated by transfection of a miR1283 mimic and strengthened by transfection of a miR-1283 inhibitor. MicroRNA 1283 bound to the 3' untranslated region of GADD45A and decreased the levels of GADD45A, which inhibited proliferation and promoted apoptosis in H/R-induced cardiomyocyte injury. Reintroduction of GADD45A attenuated the effect of miR-1283 on the viability and apoptosis of cardiomyocytes in H/R models. The JNK and p38 MAPK signaling pathways were regulated by the miR-283-GADD45A axis.

CONCLUSIONS The miR-1283-GADD45A axis may protect against H/R-induced cardiomyocyte injury by suppressing the JNK and p38 MAPK pathways.

INTRODUCTION Acute myocardial infarction (AMI) is a common cardiovascular disease caused by the necrosis of myocardial cells as a result of persistent acute ischemia and hypoxia in the coronary arteries. ${ }^{1}$ Of note, reperfusion therapy is the key to improve the prognosis of patients with AMI, but reperfusion itself increases the irreversible damage to the myocardial and coronary circulatory system, leading to an increase in the size of the infarction area, namely myocardial ischemia / reperfusion injury (MI/RI). ${ }^{2}$ Myocardial injury causes serious clinical consequences, such as no-reflow phenomenon, irreversible and reversible loss of myocardial systolic function, and reperfusion arrhythmias. ${ }^{3}$ In addition, MI / RI leads to 


\section{WHAT'S NEW?}

This study focused on examining the roles and underlying regulatory mechanisms between microRNA 1283 (miR-1283) and GADD45A in cardiomyocytes injured by hypoxia / reoxygenation. Using public online datasets, we found that miR-1283 was downregulated, and GADD45A, upregulated in myocardial infarction. Moreover, the miR-1283-GADD45A axis could protect against hypoxia / reoxygenation-induced cardiomyocyte injury by suppressing the JNK and 38 MAPK pathways in vitro and providing a potential therapeutic avenue for future reperfusion treatment of myocardial ischemia.

microcirculation disorders in up to $50 \%$ of patients who received successful transluminal percutaneous coronary intervention, which strongly affects the treatment outcome and prognosis of patients with AMI. ${ }^{4}$ Therefore, the question as to how to reduce MI/RI is crucial for the treatment of AMI and has become the focus in the field of coronary artery disease.

For the past few years, there has been increasing evidence showing that microRNAs are involved in the pathophysiological processes in the cardiovascular system, such as myocardial remodeling, myocardial hypertrophy, myocardial cell apoptosis, arrhythmia, and heart failure..$^{5-8}$ Noticeably, a study in which hematoxylin-eosin and Masson stainings were used demonstrated that the suppression of microRNA 1283 (miR-1283) could enhance cardiovascular inflammation, ${ }^{9}$ suggesting a potential role of miR-1283 in cardiovascular disease. However, the effect of miR-1283 and its regulatory relationship with the downstream molecular targets in MI/RI remains unclear.

This report aimed to assess the role of miR-1283 in cardiomyocyte injury by targeting GADD 45A using an in vitro hypoxia / reoxygenation $(\mathrm{H} / \mathrm{R})$ model. The obtained results revealed that transfection with a miR-1283 mimic attenuated H/R-induced myocardial cell injury, as evidenced by elevation of cell viability and suppression of cell apoptosis, which might have been accomplished by targeting GADD $45 \mathrm{~A}$ and inactivating the p38 MAPK and JNK signaling pathways.

METHODS Public database analysis The microarray datasets of patients with AMI and normal controls were downloaded from the Gene Expression Omnibus (GEO, http://www.ncbi.nlm. nih.gov/geo/) database. The GSE66360 dataset (https://www.ncbi.nlm.nih.gov/geo/query/acc. cgi) was contributed by Muse et al, ${ }^{10}$ who isolated circulating endothelial cells from patients with AMI $(n=49)$ and healthy controls $(n=50)$ and measured gene expression using the HG-133U_ PLUS_2 microarray. The GSE61741dataset including microRNA profiles (https://www.ncbi. nlm.nih.gov/geo/query/acc.cgi), contributed by Keller et al, ${ }^{11}$ is a complete microRNA repertoire according to miRNAse V12-14, containing data of 94 normal controls, 15 long-lived individuals, and 940 patients with AMI. Of note, the level of each microRNA was measured in at least 7 replicates. ${ }^{11}$ In the GSE61741 dataset, 94 healthy controls and 62 patients with myocardial infarction were included in this study. All differentially expressed genes (DEGs) with adjusted $P<0.05$ and $\left|\log _{2} \mathrm{FC}\right|>1$ were identified using the limma R package (Bioconductor, Boston, Massachusetts, United States). Gene Set Enrichment Analysis (GSEA, Cambridge, Massachusetts, United States), a freely available online software, ${ }^{12}$ was used to identify significant gene sets that were differentially enriched in MI, provided that the $P$ value was less than 0.05 and the false discovery rate was lower than 0.25 . No ethical approval was required for this study.

Myocardial ischemia model Human embryonic cardiomyocytes (resource no., 3111C0001CCC000177, CCC-HEH-2) from a 11-week-old male embryo tissue were acquired from Cell Resource Center of Institute of Basic Medicine, Chinese Academy of Medical Sciences (Beijing, China). To establish an in vitro myocardial H/R model, the cells were incubated in an anoxic incubator for 6 hours with $95 \% \mathrm{~N}_{2}$ and $5 \% \mathrm{CO}_{2}$ at $37^{\circ} \mathrm{C}$ after converting the Dulbecco modified Eagle medium into serum-free medium. Next, the reoxygenation process was induced in a normal incubator for 24-hour cultivation with $95 \%$ air and $5 \% \mathrm{CO}_{2}$ at $37^{\circ} \mathrm{C}$. The number of passages between thawing and use in the experiments was greater than 2 and fewer than 5 .

Real-time quantitative polymerase chain reaction analysis Using the TRIzol reagent (Thermo Fisher Scientific, Waltham, California, United States), total RNAs were isolated from cells. The PrimeScript RT Reagent Kit (Takara, Tokyo, Japan) was employed to reversely transcribe RNA into cDNA. The SYBR Premix Ex Taq II (Takara) was applied to perform real-time quantitative polymerase chain reaction (qPCR) using Applied Biosystems 7900HT (Thermo Fisher Scientific). Glyceraldehyde-3-phosphate dehydrogenase (GAPDH) was used as an internal reference. For microRNA analysis, microRNA was reversely transcribed using the MiScript Reverse Transcription kit (Qiagen, Hilden, Germany), and real-time qPCR was conducted using the MiScript SYBR-Green PCR kit (Qiagen). U6 served as control. The relative mRNA and microRNA expression was analyzed using the $\Delta \Delta$ Ct method. ${ }^{13}$

Transfection Both miR-1283 mimic and inhibitor were used to overexpress and silence miR1283 expression, respectively. A miR-1283 mimic is small, chemically modified, double-stranded RNA that mimics endogenous miR-1283 and 
enables the functional analysis of miR-1283 by upregulation of its activity. A miR-1283 inhibitor is small, chemically modified, single-stranded RNA, designed to specifically bind to and inhibit the endogenous miR-1283 molecule and enable the functional analysis of miR-1283 by downregulation of its activity. Small interfering (si) RNAs targeting GADD $45 \mathrm{~A}$ and plasmid pcDNA3.1-GADD45A were used to implement the loss- and gain-of-function of GADD45A analyses, respectively. The synthetic miR-1283 mimic/inhibitor, negative control (NC), pcDNA3.1 empty vector, pcDNA3.1-GADD45A, and siRNAs were purchased from GenePharma, Co. (Shanghai, China). The sequence of siRNA for GADD45A (si-GADD45A) was 5'-TTGCCGGGAAAGTCGCTA-3', and the sequence of the corresponding control was 5'-AATTCTCCGAACGTGTCACGT-3'. Before transfection, the cells were incubated for 24 hours in the Dulbecco modified Eagle medium, and then transfected with the corresponding vectors or siRNAs using Lipofectamine 3000 Transfection Reagent (Invitrogen, Carlsbad, California, United States) according to the manufacturer's instructions. After 24-hour incubation, the transfected cells were harvested for quantitative real-time PCR or Western blot.

Cell Counting Kit $\mathbf{8}$ assay Cell viability was evaluated using the Cell Counting Kit 8 (CCK-8) assay. After 24-hour transfection, cells were digested and counted with a cell counter to prepare cell suspension. Then, cells at a density of $1 \times 10^{3}$ cells per well were seeded in 96-well plates and cultured with $5 \% \mathrm{CO}_{2}$ at $37^{\circ} \mathrm{C}$ for $0,24,48$, and 72 hours to detect the corresponding proliferative capacity. Before detection, $10 \mu$ l of the CCK-8 reagent (Beyotime, Jiangsu, China) was added into each well. After incubation for another 1.5 hours at $37^{\circ} \mathrm{C}$, the optical density of each well was tested with a microplate reader at $450 \mathrm{~nm}$.

Cell apoptosis assay Cell apoptosis was determined using flow cytometry assay. After 24-hour transfection, the cells were collected and washed by precooled phosphate-buffered saline. Upon removing the supernatant, the cells were resuspended by $1 \times$ binding buffer to adjust cell density into $1-5 \times 10^{6} / \mathrm{ml}$. Next, cell suspension $(100 \mu \mathrm{l})$ was permitted to react with $5 \mu \mathrm{l}$ of annexin $\mathrm{V} /$ fluorescein isothiocyanate (Beyotime, Jiangsu, China) and $10 \mu$ lof propidium iodide in the darkroom for 5 minutes. Subsequently, fluorescein isothiocyanate and propidium iodide signals were tested by a flow cytometer (Becton Dickinson Biosciences, Inc., Franklin, New Jersey, United States).

Luciferase reporter assay The TargetScan software (Whitehead Institute for Biomedical Research, Cambridge, Massachusetts, United States) served to determine the binding sites between miR-1283 and the 3'UTR of GADD45A. The wild- or mutant-type of GADD45A 3'UTR, known as Wt-GADD45A or Mut-GADD45A, were generated and inserted into the pmiR-RB-REPORT plasmids (Invitrogen). Those plasmids were cotransfected with a miR-1283 mimic/inhibitor / NC using the Lipofectamine 3000 reagent (Invitrogen). After 48-hour transfection, luciferase activity was tested using the Dual-Luciferase Reporter Assay system (Promega, Madison, Wisconsin, United States).

Western blot The radioimmunoprecipitation assay buffer containing a proteinase inhibitor was used to extract proteins from cells according to the manufacturer's protocol. Samples $(20 \mu \mathrm{g})$ were separated by $10 \%$ sodium dodecyl sulfatepolyacrylamide gel electrophoresis and then transferred onto polyvinylidene fluoride membranes. Next, these membranes were blocked in $5 \%$ skim milk for an hour and incubated with primary antibodies at $4{ }^{\circ} \mathrm{C}$ for 16 hours and secondary antibodies for an hour. Enhanced Chemiluminescence Plus with a bioimaging system (Invitrogen) was used to visualize the immunoreactive protein bands, which were quantified by the ImageJ 1.44 software (National Institutes of Health, Bethesda, Maryland, United States). The following antibodies were employed in this study: anti-GADD45A (1:1000, AF6954), anti-p38 MAPK (1:1000, AF7668), anti-phospho-p38 MAPK (1:1000, AF5884), anti-JNK (1:1000, AJ518), antiphospho-JNK (1:1000, AF5860), anti-GAPDH (1:1000, AF1186), and anti-rabbit horseradish peroxidase-conjugated secondary antibodies (1:1000, A0208). The same membranes were stripped in a stripping buffer (P0025N, Beyotime, Jiangsu, China) for 15 minutes at room temperature and reprobed with an anti-GAPDH antibody used as a loading control. All these antibodies were acquired from Beyotime Biotechnology.

Statistical analysis Data were expressed as mean (SD). All experiments were performed in triplicate. Normal distribution testing was performed with the Kolmogorov-Smirnov test before comparing the study groups. The $t$ test was used to compare double groups, while multiple comparison was determined by 1-way analysis of variance followed by the Dunnett or Bonferroni post hoc test. All statistical analyses were performed using the GEOquery package of the R 3.6.0 software (R core team, Auckland, New Zealand) and the GraphPad Prism 6.0 software (La Jolla, California, United States). A P value less than 0.05 was considered significant.

RESULTS Identification of GADD45A and miR-1283 in myocardial ischemia Initially, a total of 409 genes were identified to be differentially expressed based on the cutoff 

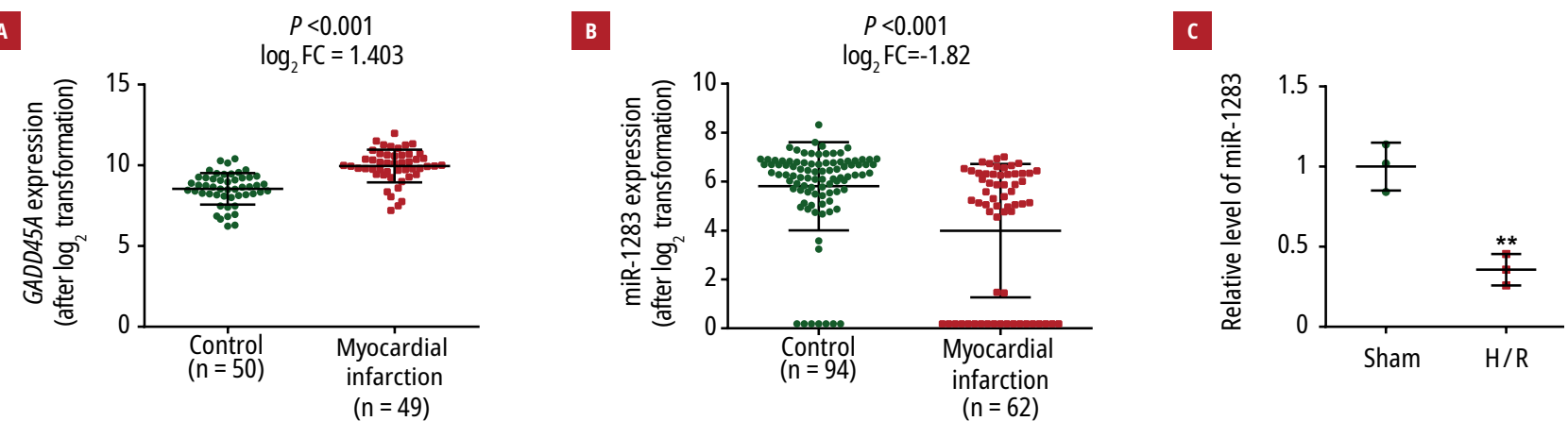

FIGURE 1 Upregulation of GADD45A and downregulation of microRNA 1283 (miR-1283) in myocardial infarction: A - expression of GADD45A in myocardial infarction samples and normal control samples (data from GSE66360); B - expression of miR-1283 in myocardial infarction samples and normal control samples (data from GSE61741); C - expression of miR-1283 in cardiomyocytes, measured by real-time quantitative polymerase chain reaction after hypoxia / reoxygenation $(\mathrm{H} / \mathrm{R})$ treatment. The double asterisk indicates $P<0.01$.

criteria of the adjusted $P$ value less than 0.05 and the absolute $\log _{2} \mathrm{FC}$ greater than or equal to 1 from the GSE66360 AMI dataset, of which 323 were upregulated and 86 were downregulated (Supplementary material, Table S1). Then, the GSEA analysis of those 409 DEGs revealed that the DEGs were remarkably enriched in 42 meaningful signaling pathways (Supplementary material, Table S2). Those signaling pathways were ranked using the normalized enrichment score and the MAPK signaling pathway ranked first with a normalized enrichment score of 1.97 (Supplementary material, Table S2 and Figure S1A). Moreover, 48 DEGs were annotated to the MAPK signaling pathway (Supplementary material, Table S3). Furthermore, 13 overlapping genes were obtained between the 323 upregulated genes and 48 MAPK-related genes, including IL1R2, IL1B, FOS, CD14, DDIT3, MAP3K8, JUN, GADD45A, GADD45ß, TNF, DUSP4, DUSP6, DUSP1 (Supplementary material, Figure S1B). Due to the limited literature on GADD45A in $\mathrm{MI}$ and the upregulation of GADD45A reported in heart failure ${ }^{14}$ GADD $45 A$ was selected as the research target, and its high expression in AMI compared with normal control $(P<0.01)$ is presented in FIGURE $1 A$.

The TargetScan software was used to predict the upstream microRNAs that could bind to the 3' UTR of GADD45A and 189 microRNAs were obtained. According to the GSE61741 dataset, 85 upregulated and 52 downregulated microRNAs were acquired (Supplementary material, Table S4). Those downregulated microRNAs and the predicted 189 microRNAs were analyzed by the Venn diagram (Supplementary material, Figure S1C) and 4 microRNAs were intersected, namely hsa-miR-524-5p, hsa-miR-520d-5p, hsa-miR-1283, and hsa-miR-518a-5p. Based on the rankings by $\log _{2} \mathrm{FC}$ values and the status of the published reports, hsa-miR-1283 was selected for further analysis. The downregulation of miR-1283 in MI compared with normal control $(P<0.01)$ is shown in FIGURE $1 B$ and in
Supplementary material (Table S4). An in vitro MI model was established using human embryonic cardiomyocytes treated with $\mathrm{H} / \mathrm{R}$. As expected, miR-1283 was notably downregulated in the $\mathrm{H} / \mathrm{R}$ group compared with the sham group $(P<0.01$; FIGURE 1C), which suggested that miR-1283 might participate in the progression of cardiomyocytes under $\mathrm{H} / \mathrm{R}$ conditions.

MicroRNA 1283 promotes proliferation and inhibits apoptosis in cardiomyocytes after hypoxia/reoxygenation treatment The CCK-8 assays revealed that $\mathrm{H} / \mathrm{R}$ reduced cell viability compared with the sham group $(P<0.01$; Supplementary material, Figure $S 2 A)$ at 48 - and 72-hour timepoints. Importantly, compared with the H / R group or H / R + NC group, a miR-1283 mimic notably enhanced cardiomyocyte viability after stimulation by $\mathrm{H} / \mathrm{R}$, whereas a miR-1283 inhibitor decreased cell viability following $H$ / $R$ treatment $(P<0.05$; Supplementary material, Figure $S 2 A$ ). Of note, due to the lack of difference between $\mathrm{H} / \mathrm{R}$ and $\mathrm{H} / \mathrm{R}+\mathrm{NC}$ groups in terms of cell proliferation, the $\mathrm{H} / \mathrm{R}$ blank was used as control in the following experiments to determine the effects of a miR-1283 mimic/inhibitor on cell apoptosis after H / R treatment. The data obtained with the use of flow cytometry assays revealed that the percentage of apoptosis was remarkably elevated in $\mathrm{H} / \mathrm{R}$-damaged cardiomyocytes compared with the sham control ( $P<0.01$; Supplementary material, Figure $S 2 B$ and $S 2 C$ ), and the increased cell apoptotic capacity was notably reinforced by miR-1283 inhibitor transfection $(P<0.01$; Supplementary material, Figure $S 2 B$ and $S 2 C$ ) yet reversed by miR-1283 mimic transfection $(P<0.01$; Supplementary material, Figure $S 2 B$ and $S 2 C$ ). Thus, we deduced that the increase in cell activity induced by miR-1283 may be partially attributed to the reduction in apoptosis under $\mathrm{H} / \mathrm{R}$ conditions. Altogether, the abovementioned data implied that miR-1283 can ameliorate cardiomyocyte injury induced by $\mathrm{H} / \mathrm{R}$. 
3' UTR of GADD45A mRNA (Wt)

miR-1283

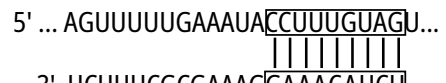

3' UCUUUCGCGAAAGGGAACAUCU...

3' UTR of GADD45A mRNA (Mut) 5' ... AGUUUUUGAAAUAUACCGACGA ...

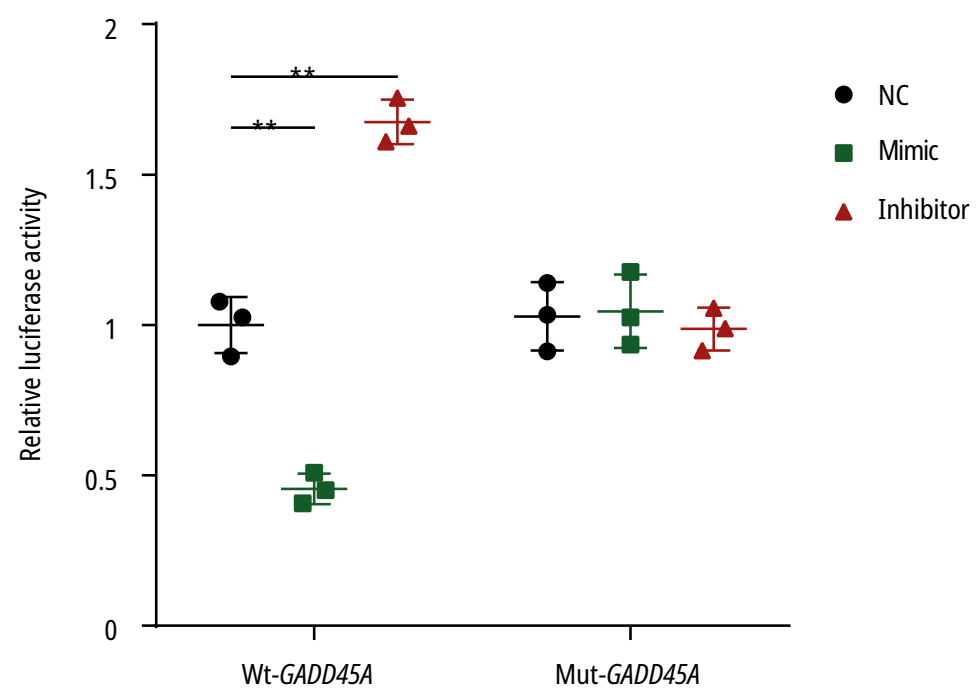

FIGURE 2 The potential binding sites between microRNA 1283 (miR-1283) and GADD45A were determined with the use of the TargetScan software. The luciferase reporter activity of pmiR-RB-REPORT plasmids carrying Wt-GADD45A and Mut-GADD45A transfected with negative control (NC) and a miR-1283 mimic or inhibitor were measured using luciferase reporter assays. The double asterisk indicates $P<0.01$.

Abbreviations: NC, negative control; UTR, untranslated region

MicroRNA 1283 directly targets GADD45A and negatively regulates its expression in cardiomyocytes The potential binding sites between miR-1283 and GADD45A are presented in FIGURE2. The results shown in FIGURE 2 indicated that the luciferase activity of the pmiR-RB-REPORT plasmid carrying Wt-GADD45A was remarkably inhibited in the miR-1283 mimic group and elevated in the miR-1283 inhibitor group compared with the NC group $(P<0.01)$, whereas that of the pmiR-RB-REPORT plasmid carrying Mut-GADD45A was not changed either in the miR-1283 mimic group or the inhibitor group, which suggested that miR-1283 targeted GADD 45A by binding to its 3' UTR sequence. Furthermore, data from real-time qPCR and Western blot analyses revealed that mRNA and the protein level of GADD45A in cardiomyocytes were reduced or enhanced after transfection with si-GADD45A or pcDNA3.1-GAD$D 45 A$, respectively, compared with the appropriate control $(P<0.01$; Supplementary material, Figure S3A-S3E). Moreover, a miR-1283 mimic/inhibitor notably inhibited or elevated the expression of GADD45A, respectively, at both mRNA and protein levels, while both the upregulation and downregulation of miR-1283 and GADD45A in cardiomyocytes could attenuate that reduction or elevation, respectively $(P<0.01$; Supplementary material,
Figure $S 3 A-S 3 E$ ). To sum up, all these data demonstrated that GADD $45 A$ acts as a downstream target gene of miR-1283.

GADD45A contributes to the effect of microRNA 1283 on hypoxia/reoxygenation-induced cell viability and apoptosis Compared with the NC group, GADD45A-knockdown led to an increase in the optical density values and resulted in a notable decrease in the apoptotic rate after $\mathrm{H} / \mathrm{R}$ damage in cardiomyocytes $(P<0.05$; FIGURE3B and 3D; Supplementary material, Figure $S 4 B$ ). On the other hand, the optical density values were considerably decreased, whereas the apoptotic rate rose in cardiomyocytes transfected with pcDNA3.1-GAD$D 45 A$ under $\mathrm{H} / \mathrm{R}$ conditions $(P<0.05$; FIGURE $3 \mathrm{~A}$ and 3c; Supplementary material, Figure $S 4 A$ ). Thus, the knockdown of GADD45A might mitigate cardiomyocyte injury derived from $\mathrm{H} / \mathrm{R}$.

To verify whether GADD45A is involved in the effects of miR-1283 on proliferation and apoptosis, rescue CCK-8 and flow cytometry analyses were implemented. Surprisingly, under H / R conditions, the increase or decrease in cardiomyocyte proliferation or apoptosis, caused by a miR-1283 mimic, could be, at least partially, reversed by the introduction of pcDNA3.1-GADD45A $(P<0.05$; FIGURE 3A and 3C; Supplementary material, Figure $S 4 A)$. On the contrary, miR-1283 inhibitor-induced reduction of cell proliferation and enhancement of cell apoptosis were reversed by GADD $45 A$ knockdown after $\mathrm{H} / \mathrm{R}$ treatment $(P<0.01$; FIGURE 3 B and 3D; Supplementary material, Figure S4B). In short, these data implied that GADD45A blocks the protective effects of miR-1283 in cardiomyocytes during $\mathrm{H} / \mathrm{R}$-induced injury.

The microRNA 1283-GADD45A axis mediates cardiomyocyte injury induced by hypoxia / reoxygenation through the JNK and p38 MAPK signaling pathways Initially, GSEA showed that the MAPK signaling pathway was upregulated in AMI according to the gene expression profile. Moreover, GADD45A was significantly related to that pathway. Thus, we performed Western blot analysis to determine the effects of the miR-1283-GADD45A axis on the MAPK signaling pathway in cardiomyocytes after $\mathrm{H} / \mathrm{R}$ treatment. The results demonstrated that the protein levels of phospho-p38 MAPK and phospho-JNK were reduced or elevated by a miR-1283 mimic or inhibitor, respectively, compared with the appropriate NC group under $\mathrm{H} / \mathrm{R}$ conditions $(P<0.01$; FIGURE $4 \mathrm{~A}$ and $4 \mathrm{~B})$. However, the over- or underexpression of GADD 45A in cardiomyocytes following $\mathrm{H} / \mathrm{R}$ treatment caused enhancement or decrement, respectively, on the protein levels of those 2 markers compared with the appropriate $\mathrm{H} / \mathrm{R}+\mathrm{NC}$ group $(P<0.01$; FIGURE $4 \mathrm{~A}$ and $4 \mathrm{~B})$. Notably, the decrease or increase in the protein 

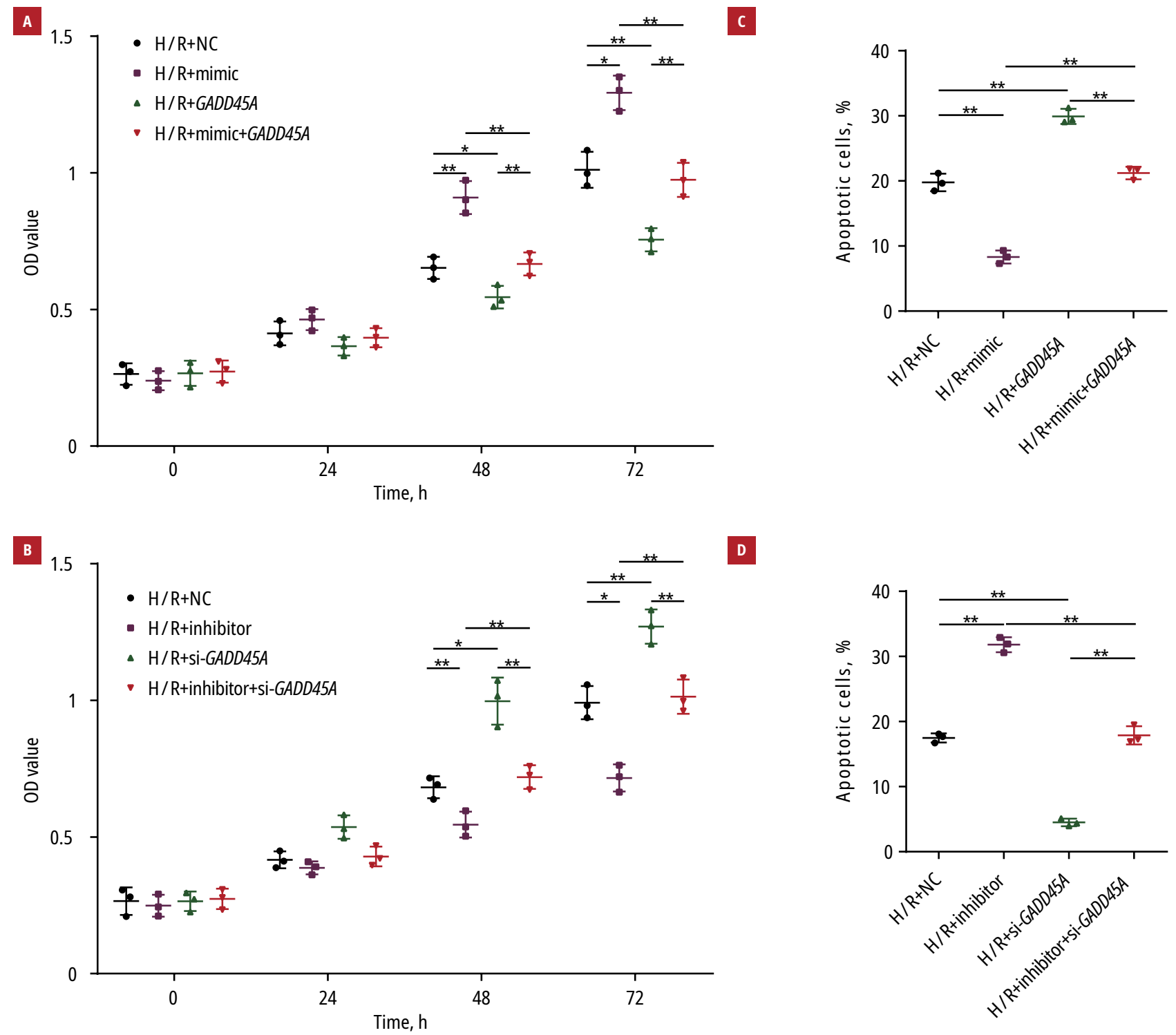

D

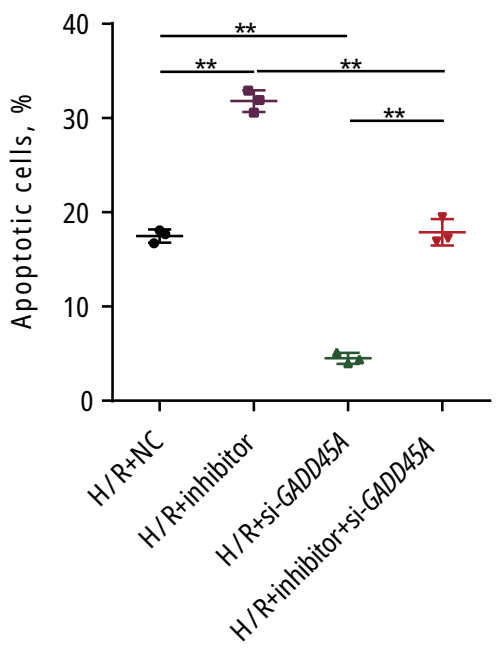

FIGURE 3 Effect of the microRNA 1283 (miR-1283)-GADD45A axis on cardiomyocyte proliferation and apoptosis in hypoxia / reoxygenation (H/R) models: A, B - optical density (OD) values of cardiomyocytes in H/R models transfected with negative control (NC), a miR-1283 mimic or inhibitor, pCDNA3.1-GADD45A/ si-GADD45A, and miR-1283 mimic + pcDNA3.1-GADD45A/ miR-1283 inhibitor + si-GADD45A were measured using Cell Counting Kit 8 (CCK-8) assays at 0, 24, 48, and 72 hours. C, D - cell apoptosis was determined by flow cytometry in each group under $\mathrm{H} / \mathrm{R}$ conditions. The asterisk indicates $P<0.05$, and the double asterisk, $P<0.01$.

levels of phospho-p38 MAPK and phospho-JNK caused by a miR-1283 mimic or inhibitor, respectively, can be abrogated by cotransfection with pcDNA3.1-GADD45A/si-GADD45A in $\mathrm{H} / \mathrm{R}$-treated cells $(P<0.01$; FIGURE $4 \mathrm{~A}$ and $4 \mathrm{~B})$. Interestingly, no obvious changes were observed on the protein levels of p38 MAPK and JNK in all groups. Thus, we inferred that the miR-1283-GADD45A axis represses the JNK and $\mathrm{p} 38$ MAPK signaling pathways to exert its protective role in $\mathrm{H} / \mathrm{R}$-induced cardiomyocyte injury.

DISCUSSION A growing body of evidence has demonstrated that numerous microRNAs exert a cardioprotective effect, which indicates that they play a pivotal role in MI/ RI. ${ }^{15-17}$ It has been proposed that miR-1283 participated in the development of multiple diseases. For example, miR1283 introduction remarkably suppressed glioma cell proliferation and invasion. ${ }^{18}$ Inhibition of miR-1283 promoted vascular endothelial cell injury by targeting activating transcription factor $4 .{ }^{19}$ Additionally, it may be involved in essential hypertension..$^{20}$ However, the role of miR-1283 with respect to its function in protecting against ischemic injury has not been reported yet. The present study is the first to reveal that the introduction of miR-1283 increased cell viability and suppressed apoptosis, whereas the inhibition of miR-1283 yielded the opposite outcomes, which suggests that miR-1283 contributes to protecting cardiomyocytes against $\mathrm{H} / \mathrm{R}$-related injury.

MicroRNAs are involved in the regulation of vital activity by binding to the 3' UTR of several 
A

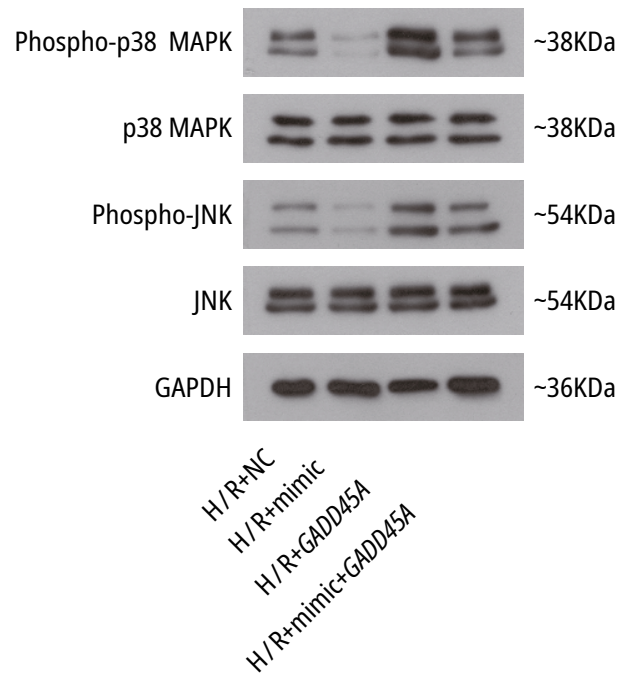

B
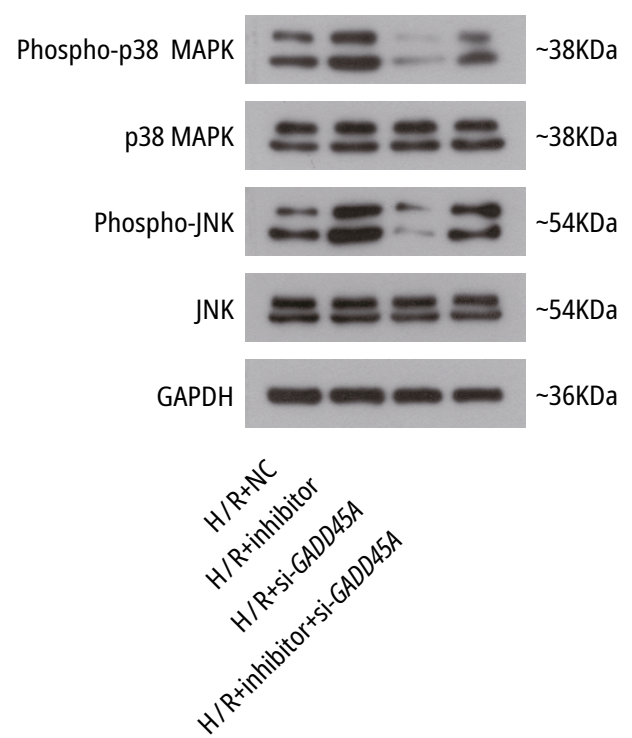

- $\mathrm{H} / \mathrm{R}+\mathrm{NC}$

- $\mathrm{H} / \mathrm{R}+G A D D 45 A$

- $H / R+m i m i c$

- $\mathrm{H} / \mathrm{R}+$ mimic $+G A D D 45 A$
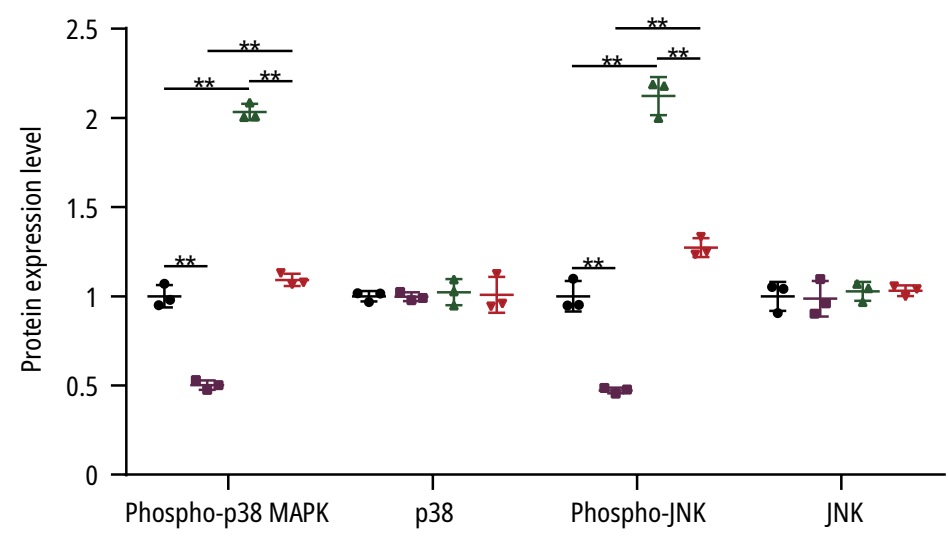

- $\mathrm{H} / \mathrm{R}+\mathrm{NC}$

- $\mathrm{H} / \mathrm{R}+\mathrm{si}-G A D D 45 A$

- $\mathrm{H} / \mathrm{R}+$ inhibitor

- $\mathrm{H} / \mathrm{R}+$ inhibitor+si-GADD45A

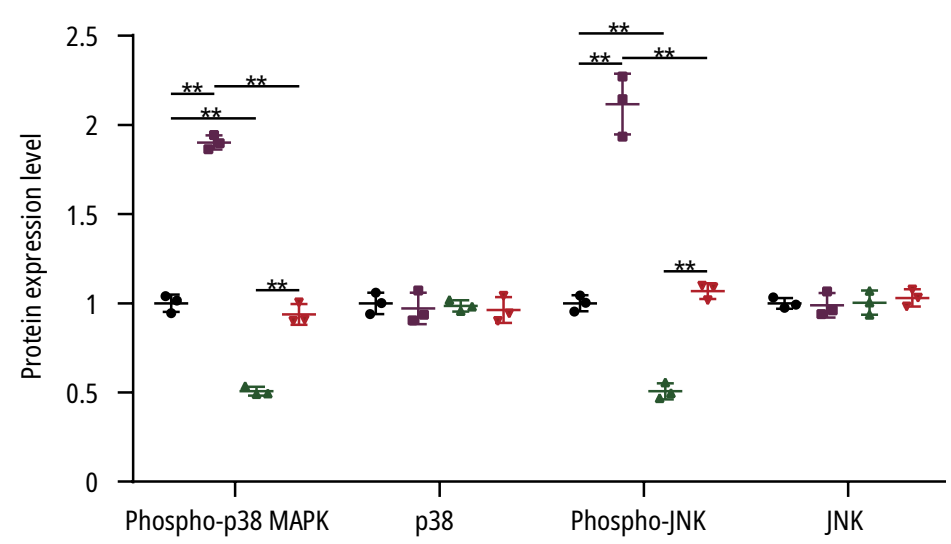

FIGURE 4 The microRNA 1283-GADD45A axis affected the protein levels of MAPK signaling pathway-related markers: A, B - Western blot assays were used to examine the levels of relative protein in the MAPK signaling pathway in each group. Protein levels are in the left panels, and their quantification, normalized to the GAPDH level, is presented on the right. The double asterisk indicates $P<0.01$.

Abbreviations: H/R, hypoxia / reoxygenation; others, see FIGURE 2

targets. ${ }^{21}$ In this study, GADD $45 A$ was identified to be a downstream target of miR-1283 and was upregulated in MI. Of note, the regulatory relationship between miR-1283 and GADD45A was first revealed in this report. As the first member of the GADD45 family, which is a known group of stress sensors, GADD $45 A$ plays a crucial regulatory role in various cellular functions such as DNA repair, cell cycle regulation and senescence, and genotoxic stress response. ${ }^{22}$ Also, numerous studies have demonstrated that GADD $45 A$ is associated with the malignant biologic behavior of some tumors, including pancreatic, breast, and esophageal cancers. ${ }^{23-26}$ In cardiac disease, $G A D D 45 A$, as a target gene of miR-148b-3p, was upregulated in patients with heart failure with mitral regurgitation compared with normal controls. ${ }^{14}$ However, studies have rarely investigated the activity of GADD45A in cardiomyocyte injury, especially its regulatory relationship with the other upstream microRNAs.

As a p53 target gene, GADD45A is activated by p53 in response to cellular DNA damage..$^{27,28}$ Activated GADD $45 A$ regulates cell cycle arrest and DNA damage repair and induces apoptosis. ${ }^{29}$ In this report based on the public GEO database, GADD45A was highly expressed in tissues affected by AMI, so we inferred that $G A D$ $D 45 A$ may play a role in $\mathrm{MI} / \mathrm{RI}$. In fact, we also observed that GADD45A exerted antiproliferative and proapoptotic effects on $\mathrm{H} / \mathrm{R}$-damaged cardiomyocytes, which indicated that GADD $45 \mathrm{~A}$ 
knockdown may be helpful in protecting cardiomyocytes against reperfusion injury.

MAPK signaling pathway-related proteins can be activated by ischemia / reperfusion (I / R) and may play crucial roles in the evolution of ischemic injury. ${ }^{30}$ As a key member of the MAPK signaling pathway, p38 MAPK is a significant player in inflammation, tumor development, I/ R organ injury, myocardial remodeling, etc. ${ }^{31}$ Numerous studies have shown that p38 MAPK is involved in myocardial cell apoptosis, which mainly occurs during reperfusion and constitutes one of the main causes of myocardial injury. ${ }^{32,33}$ A study demonstrated that excessive reactive oxygen species were formed at an early stage of reperfusion, which activated p38 MAPK and eventually led to myocardial cell apoptosis. ${ }^{34}$ Besides, another member of the MAPK family, JNK, is a major signal transduction molecule in cells. In the postinjury stress response, JNK can induce apoptosis and is closely related to cell proliferation. ${ }^{35}$ A report has shown that the JNK signal transduction pathway is closely linked to MI. ${ }^{36}$ Regarding MI/RI, some studies found that activated JNK could be detected within $10 \mathrm{~min}$ utes after injury, which suggests that JNK may play a role at an early stage of injury. ${ }^{37}$ Our findings demonstrated that the protective effect of the miR-1283-GADD45A axis on cardiomyocytes treated by $\mathrm{H} / \mathrm{R}$ was achieved through regulating the JNK and p38 MAPK signaling pathways.

Limitations Several limitations of this study need to be acknowledged. First of all, our findings were based on individual cell lines and it would be of value to reproduce the results on a different clone of cells. Second, this work was a foundational study examining the functional role of the miR-1283-GADD45A axis in human embryonic cardiomyocytes. It would be more persuasive to use an internal system closer to human MI. Third, in our view, the miR1283-GADD45A axis may be strongly correlated with ERK, which is an important factor of the MAPK signaling pathway. Another study has also documented that GADD45A was upregulated by the MAPK-ERK pathway following cisplatin treatment for human melanoma. ${ }^{38}$ Further analysis including ERK needs to be considered.

Conclusions The current report revealed that miR-1283 could protect cardiomyocytes against $\mathrm{H} / \mathrm{R}$ damage through targeting GADD45A by regulating the JNK and p38 MAPK signaling pathways, which was manifested in enhancing cell viability and reducing apoptosis. These findings will help us to find more effective ways to protect myocardial cells against I / R injury.

\section{SUPPLEMENTARY MATERIAL}

Supplementary material is available at www.mp.pl/kardiologiapolska.

\section{ARTICLE INFORMATION}

ACKNOWLEDGMENTS This work was supported by the Construction Project of Heilongjiang Province's Northern Medicine and Functional Food Advantage Specialty Subject, Biology Subject Team of Jiamusi University (grant no. jdxktd-2019003; to PZ).

\section{CONFLICT OF INTEREST None declared.}

OPEN ACCESS This is an Open Access article distributed under the terms of the Creative Commons Attribution-NonCommercial-NoDerivatives $4.0 \mathrm{In}$ ternational License (CC BY-NC-ND 4.0), allowing third parties to download articles and share them with others, provided the original work is properly cited, not changed in any way, distributed under the same license, and used for noncommercial purposes only. For commercial use, please contact the journal office at kardiologiapolska@ptkardio.pl.

HOW TO CITE Liu C, Liu H, Sun Q, Zhang P. MicroRNA 1283 alleviates cardiomyocyte damage caused by hypoxia / reoxygenation via targeting GADD45A and inactivating the JNK and p38 MAPK signaling pathways. Kardiol Pol. 2021; 79: 147-155. doi:10.33963/KP.15696

\section{REFERENCES}

1 Du H, Hao J, Liu F, et al. Apigenin attenuates acute myocardial infarction of rats via the inhibitions of matrix metalloprotease-9 and inflammatory reactions. Int J Clin Exp Med. 2015; 8: 8854-8859.

2 Frank A, Bonney M, Bonney S, et al. Myocardial ischemia reperfusion injury: from basic science to clinical bedside. Semin Cardiothorac Vasc Anesth. 2012; 16: $123-132$.

3 Moens AL, Claeys MJ, Timmermans JP, et al. Myocardial ischemia/reperfusion-injury, a clinical view on a complex pathophysiological process. Int J Cardiol. 2005; 100: $179-190$.

4 Niccoli G, Scalone G, Lerman A, et al. Coronary microvascular obstruction in acute myocardial infarction. Eur Heart J. 2016; 37: 1024-1033.

5 Schulte C, Karakas M, Zeller T. microRNAs in cardiovascular disease - clinical application. Clin Chem Lab Med. 2017; 55: 687-704.

6 Li Q, Yang J, Zhang J, et al. Inhibition of microRNA-327 ameliorates ischemia/ reperfusion injury-induced cardiomyocytes apoptosis through targeting apoptosis repressor with caspase recruitment domain. J Cell Physiol. 2020; 235: 3753-3767.

7 Liu JY, Shang J, Mu XD, et al. Protective effect of down-regulated microRNA-27a mediating high thoracic epidural block on myocardial ischemia-reperfusion injury in mice through regulating $A B C A 1$ and $\mathrm{NF}-\mathrm{kB}$ signaling pathway. Biomed Pharmacother. 2019; 112: 108606-108615.

8 Yuan L, Fan L, Li Q, et al. Inhibition of miR-181b-5p protects cardiomyocytes against ischemia/reperfusion injury by targeting AKT3 and PI3KR3. J Cell Biochem. 2019; 120: 19647-19659.

9 He L, Yuan J, Xu Q, et al. miRNA-1283 regulates the PERK/ATF4 pathway in vascular injury by targeting ATF4. PLOS One. 2016; 11: 171-187.

10 Muse ED, Kramer ER, Wang $H$, et al. A whole blood molecular signature for acute myocardial infarction. Sci Rep. 2017; 7: 12268.

11 Keller A, Leidinger P, Vogel B, et al. miRNAs can be generally associated with human pathologies as exemplified for miR-144. BMC Med. 2014; 12: 224.

12 Subramanian A, Tamayo P, Mootha VK, et al. Gene set enrichment analysis: a knowledge-based approach for interpreting genome-wide expression profiles. Proc Natl Acad Sci USA. 2005; 102: 15545-15550.

13 Livak KJ, Schmittgen TD. Analysis of relative gene expression data using real-time quantitative PCR and the 2(-Delta Delta C(T)) Method. Methods. 2001; 25: $402-408$.

14 Chen MC, Chang TH, Chang JP, et al. Circulating miR-148b-3p and miR-409-3p as biomarkers for heart failure in patients with mitral regurgitation. Int J Cardiol. 2016; 222: 148-154

15 Tan H, Qi J, Fan BY, et al. MicroRNA-24-3p attenuates myocardial ischemia/ reperfusion injury by suppressing RIPK1 expression in mice. Cell Physiol Biochem. 2018; 51: 46-62.

16 Yang N, Wu L, Zhao Y, et al. MicroRNA-320 involves in the cardioprotective effect of insulin against myocardial ischemia by targeting survivin. Cell Biochem Funct. 2018; 36: 166-171.

17 Zhao YB, Zhao J, Zhang LJ, et al. MicroRNA-370 protects against myocardial ischemia/reperfusion injury in mice following sevoflurane anesthetic preconditioning through PLIN5-dependent PPAR signaling pathway. Biomed Pharmacother. 2019; 113: 108697-108707.

18 Chen H, Zhang Y, Su H, et al. Overexpression of miR-1283 inhibits cell proliferation and invasion of glioma cells by targeting ATF4. Oncol Res. 2019; 27: 325-334.

19 He L, Yuan J, Xu Q, et al. miRNA-1283 regulates the PERK/ATF4 pathway in vascular injury by targeting ATF4. PLoS One. 2016; 11: e0159171.

20 Yang S, Gao Y, Liu G, et al. The human ATF1 rs11169571 polymorphism increases essential hypertension risk through modifying miRNA binding. FEBS Lett. 2015; 589: 2087-2093.

21 Mohr AM, Mott JL. Overview of microRNA biology. Semin Liver Dis. 2015; 35: 3-11.

22 Salvador JM, Brown-Clay JD, Fornace AJ Jr. GADD45 in stress signaling, cell cycle control, and apoptosis. Adv Exp Med Biol. 2013; 793: 1-19. 
23 Wang J, Wang Y, Long F, et al. The expression and clinical significance of GADD45A in breast cancer patients. Peer]. 2018; 6: e5344.

24 Ishiguro $\mathrm{H}$, Kimura M, Takahashi $\mathrm{H}$, et al. GADD45A expression is correlated with patient prognosis in esophageal cancer. Oncol Lett. 2016; 11: 277-282.

25 Dong $M$, Dong $Q$, Zhang $H$, et al. Expression of GADD45a and p53 proteins in human pancreatic cancer: potential effects on clinical outcomes. J Surg Oncol. 2007; 95: 332-336.

26 Han N, Yuan F, Xian P, et al. GADD45a mediated cell cycle inhibition is regulated by p53 in bladder cancer. Onco Targets Ther. 2019; 12: 7591-7599.

27 Jin S, Mazzacurati L, Zhu X, et al. GADDA45a contributes to p53 stabilization in response to DNA damage. Oncogene. 2003; 22: 8536-8540.

28 Cannell IG, Merrick KA, Morandell S, et al. A pleiotropic RNA-binding protein controls distinct cell cycle checkpoints to drive resistance of p53-defective tumors to chemotherapy. Cancer Cell. 2015; 28: 623-637.

29 Xie L, Jia L, Qu J, et al. Expression and prognostic significance of the P53-related DNA damage repair proteins checkpoint kinase 1 (CHK1) and growth arrest and DNA-damage-inducible 45 alpha (GADD45A) in human oral squamous cell carcinoma. Eur J Oral Sci. 2020; 128: 128-135.

30 Steenbergen $C$. The role of p38 mitogen-activated protein kinase in myocardial ischemia/reperfusion injury; relationship to ischemic preconditioning. Basic Res Cardiol. 2002; 97: 276-285.

31 Kim EK, Choi EJ. Compromised MAPK signaling in human diseases: an update. Arch Toxicol. 2015; 89: 867-882.

32 Guo W, Liu X, Li J, et al. Prdx1 alleviates cardiomyocyte apoptosis through ROS-activated MAPK pathway during myocardial ischemia/reperfusion injury. Int J Biol Macromol. 2018; 112: 608-615.

33 Gao R, Fang Q, Zhang $X$, et al. R5 HIV-1 gp120 activates p38 MAPK to induce rat cardiomyocyte injury by the CCR5 coreceptor. Pathobiology. 2019; 86: 274-284. 34 Yu D, Li M, Tian Y, et al. Luteolin inhibits ROS-activated MAPK pathway in myocardial ischemia/reperfusion injury. Life Sci. 2015; 122: 15-25.

35 Solinas G, Becattini B. JNK at the crossroad of obesity, insulin resistance, and cell stress response. Mol Metab. 2017; 6: 174-184.

36 Kuan CY, Whitmarsh AJ, Yang DD, et al. A critical role of neural-specific JNK3 for ischemic apoptosis. Proc Natl Acad Sci USA. 2003; 100: 15184-15189.

37 Frazier DP, Wilson A, Dougherty C], et al. PKC-alpha and TAK-1 are intermediates in the activation of c-Jun NH2-terminal kinase by hypoxia-reoxygenation. Am J Physiol Heart Circ Physiol. 2007; 292: H1675-H1684

38 Liu J, Jiang G, Mao P, et al. Down-regulation of GADD45A enhances chemosensitivity in melanoma. Sci Rep. 2018; 8: 4111-4121. 